\title{
Three-dimensional porous architectures of carbon nanotubes and graphene sheets for energy applications
}

\author{
Xuewan Wang, Gengzhi Sun and Peng Chen* \\ Division of Bioengineering, School of Chemical and Biomedical Engineering, Nanyang Technological University, Singapore, Singapore
}

Edited by:

Lain-Jong Li, Academia Sinica, Taiwan

Reviewed by:

Yumeng Shi, Singapore University of Technology and Design, Singapore

Yi-Hsien Lee, National Tsing-Hua

University, Taiwan

*Correspondence:

Peng Chen, Division of

Bioengineering, School of Chemical

and Biomedical Engineering, Nanyang

Technological University, 70 Nanyang

Drive 637457, Singapore

e-mail: chenpeng@ntu.edu.sg
Owing to their extraordinary physicochemical, electrical, and mechanical properties, carbon nanotubes (CNTs) and graphene materials have been widely used to improve energy storage and conversion. In this article, we briefly review the latest development on fabrication of 3D porous structures of CNTs or graphene sheets or their hybrids, and their applications in various energy devices including supercapacitors, (bio-) fuel cells, and lithium ion batteries.

Keywords: carbon nanotube, graphene, three-dimensional architecture, energy storage, energy conversion

\section{INTRODUCTION}

In the past decades, carbon nanotubes (CNTs) and graphen have changed the landscape of many fields in science and technology, including energy storage and conversion (Sun et al., 2011b; Liu et al., 2012; Weiss et al., 2012; Jariwala et al., 2013; Zhang et al., 2013). Although differing in structure, synthetic routes, and physicochemical properties, the two carbon allotropes share common merits for energy applications, such as good electrical conductivity, high-specific surface area, excellent mechanical strength, and flexibility. In frequent cases, integration of 1D CNT and 2D graphene materials exerts synergistic effects. Despite the great success achieved, further extending their potential, to a great extent, depends on the possibility to assemble these nanocarbon materials into macroscopic 3D architectures while preserving the intrinsic properties of individual building components.

The electrochemical electrodes used in energy devices or for other purposes are conventionally planar. Electrodes based on 3D porous nanocarbon structures can greatly improve the capacity and efficiency because of largely expanded working volume, multiplexed conduction network, 3D interfacing, or intercalation with other system components (e.g., electrolytes, reactants). However, it is non-trivial to assemble CNTs and graphene sheets into 3D architectures due to their small size as well as stacking or aggregation caused by strong hydrophobic and pi-pi interactions. In this article, we review the latest development on synthesis of macroporous CNT/graphene structures and their applications in energy devices.

\section{SYNTHESIS APPROACHES}

The $3 \mathrm{D}$ porous $\mathrm{CNT}$ and graphene structures can be readily fabricated from CNT/graphene solvent dispersions via self-assembly or substrate-based deposition, which are cost-effective and easily scalable. On the other hand, chemical vapor deposition (CVD) is able to produce $3 \mathrm{D}$ porous $\mathrm{CNT} /$ graphene monoliths, with high quality and well-defined architectures. Some other approaches have also been recently developed.

\section{D CNT STRUCTURES}

Direct deposition of dispersed CNTs onto a 3D substrate is simple and effective to construct porous architectures. For example, sponge with continuous 3D surface and good mechanical flexibility can serve as a supporting substrate. CNT-sponge composites fabricated via dip-coating method are highly porous $(98 \%$, pore size 200-500 $\mu \mathrm{m}$ ) with large specific surface area of $\sim 10^{4} \mathrm{~m}^{2} \mathrm{~m}^{-3}$ (Xie et al., 2012). The thin CNT coating layer provides the composite a conductance of $\sim 1 \mathrm{~S} \mathrm{~cm}^{-1}$. Without use of any template, self-assembled CNT foam has been made by a low temperature chemical fusion method (Figure 1) (Liu et al., 2013b). Meso/macroporosity of the CNT foam can be tuned by the amount of ammonia carbonate, which acts as the pore former. The assynthesized CNT foam can withstand a stress pressure as high as 1.39 MPa. Catalytic growth of CNT forests via CVD method is advantageous in well controlling the length, quality, and density of CNTs (Zheng et al., 2010; Zhan et al., 2011). Zhao et al. (2011) sputtered Fe catalysts on the surface of Ni foam for the growth of 3D randomly entangled CNTs. Shan et al. (2013) synthesized 3D sponge-like N-doped CNT architecture, employing the mixture of ferrocene, thiophene, and pyridine as CVD precursors. The $\mathrm{N}$ and $\mathrm{S}$ are proposed to synergistically promote the formation of "elbow" and "welded" junctions between CNTs. In addition, the diameter of N-doped multi-walled CNTs can be easily controlled by modulating thiophene concentration, which in turn influences the mechanical and electrical properties of N-doped MWCNT sponge.

\section{D GRAPHENE STRUCTURES Graphene hydrogels and aerogels}

The hydrophilic edges and hydrophobic basal planes of graphene oxide (GO) render it to be amenable to solution-based processes 
(Sun et al., 2011a). Direct gelation of GO dispersion into porous bulk structures can be achieved by freeze-drying and hydrothermal methods (Jiang et al., 2010; Xu et al., 2010; Sun et al., 2014). Recently, Xie et al. (2013) devised a modified freeze casting process. They found that the microstructures and properties

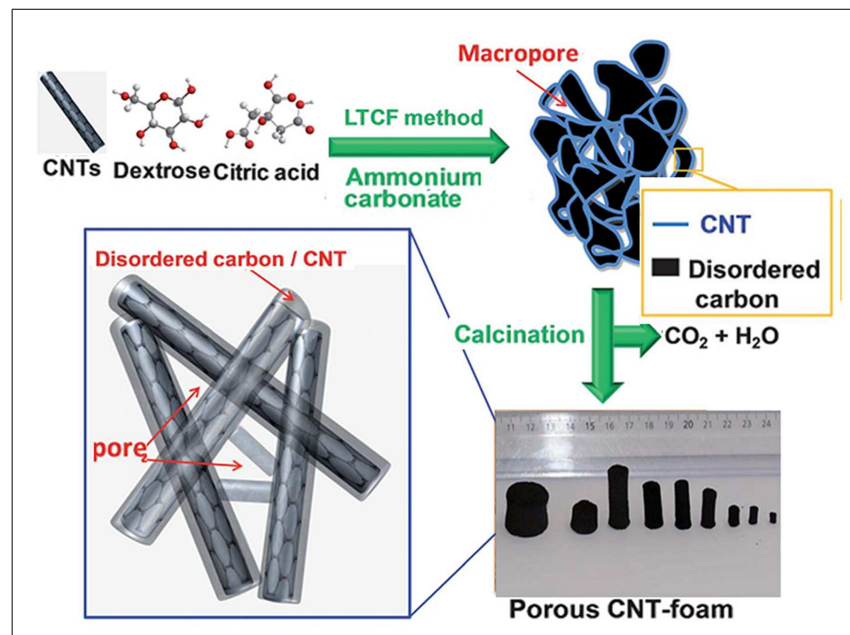

FIGURE 1 | Illustration of a low temperature chemical fusion method for the synthesis of porous CNT-foam composites. Adapted with permission from Liu et al. (2013b). Copyright 2013 Royal Society of Chemistry. of the resulting porous graphene aerogel can be widely adjusted by the freezing temperature. Liquid nitrogen freezing confers the as-synthesized aerogel an isotopic and highly elastic structure. Wan et al. covalently cross-linked GO sheets into a monolith by conjugating the epoxy groups on GO with amine groups of a gelating polymer via ring opening reaction (Xie et al., 2013). The obtained GO hydrogel is highly elastic and can be easily converted into reduced GO (rGO) hydrogel via thermal treatment. Without using any linkage additives or chemical reducing agents, Hu et al. (2013) demonstrated substrate-assisted spontaneous reduction and assembly of GO sheets into 3D network on various conductive substrates (Figure 2A). The 3D graphene structures have also been obtained by repetitive dip-coating GO sheets into sponge, followed by GO reduction and removal of sponge by heating (Figure 2B) (Yang et al., 2014). Interestingly, Peng et al. (2014) reported a chemical unzipping approach to convert CNT sponge into graphene nanoribbon aerogel (Figure 2C). The resulting aerogel inherits the $3 \mathrm{D}$ network and high porosity of CNT sponge, and gains enhanced surface area and chemical functionality. It has also been shown that further hydrazine reduction helps the recovery of mechanical properties of graphene nanoribbon aerogel impaired by unzipping process. Graphene aerogel with hierarchical porous structure has also been fabricated using a so-called "sugar-blowing" approach by carbonization of a mixture of glucose and ammonia chloride (Figure 3A) (Wang et al., 2013). Such ultralight $\left(3.0 \mathrm{mg} \mathrm{cm}^{-3}\right)$ structure possesses a good mechanical strength and is highly compressible.
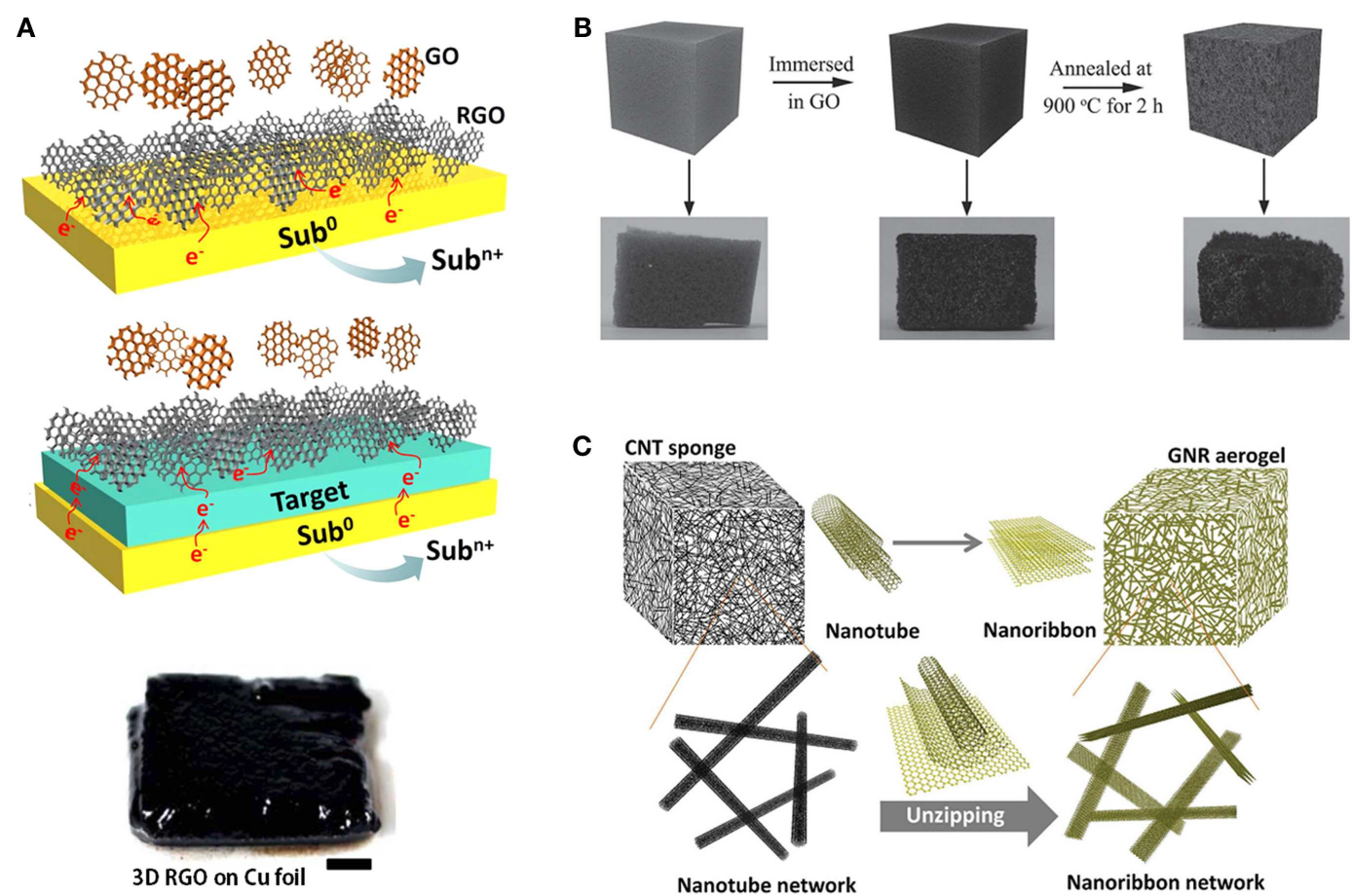

FIGURE 2 | (A) Scheme of the substrate-assisted reduction and assembly of $\mathrm{GO}$ on active metal substrate and arbitrary conductive target supported by active metal. Adapted with permission from Hu et al. (2013). Copyright 2013 Nature Publishing Group. (B) Schematic representation and photo images of the procedure for preparing sponge template graphene aerogel. Adapted with permission from Yang et al. (2014). Copyright 2014 WILEY-VCH Verlag GmbH \& Co. KGaA, Weinheim. (C) Illustration of the process in which a CNT sponge is directly converted into a GNR aerogel by unzipping method. Adapted with permission from Peng et al. (2014). Copyright 2014 WILEY-VCH Verlag GmbH \& Co. KGaA, Weinheim. 


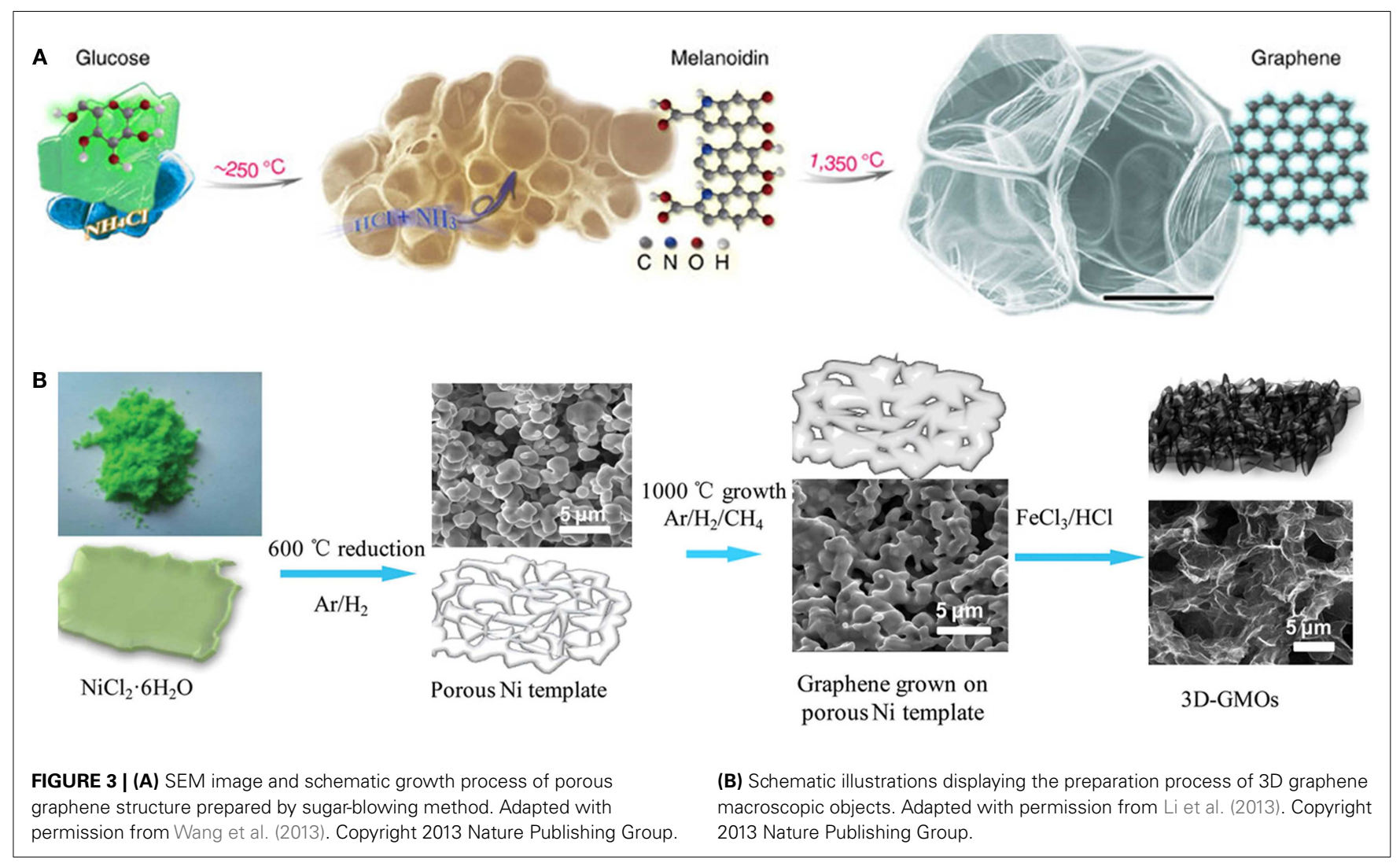

\section{CVD GROWN 3D GRAPHENE}

As first demonstrated by Chen et al. (2011), 3D defect-free graphene monolith can be obtained by CVD using $\mathrm{Ni}$ foam as the growth substrate. Such graphene foam exhibits macroporous structure $(99.7 \%$ porosity), high-specific surface area $\left(\sim 850 \mathrm{~m}^{2} \mathrm{~g}^{-1}\right)$, and high conductivity $\left(\sim 10 \mathrm{~S} \mathrm{~cm}^{-1}\right)$. The Ni foam can be subsequently removed by acid, leaving light weighted and free-standing graphene foam (Dong et al., 2012f). Recently, Li et al. (2013) fabricated microporous 3D graphene foams via a rapid CVD process using nickel chloride hexahydrate as the catalyst precursor (Figure 3B). Prolonging the growth time enhances the thickness and strength of graphene layers. Using plasma enhanced CVD process, dense vertically oriented graphene sheets were grown on Ni foam, offering a large surface area (Ren et al., 2014).

\section{D CNT-GRAPHENE HYBRIDS}

CNT and graphene possess a plethora of different characteristics in structural and physicochemical properties. To enjoy the synergistic effects of these two graphitic allotropes, 3D CNT/graphene hybrid architectures have been prepared by hydrothermal treatment of CNT and graphene solution mixture (You et al., 2013). In this composite, the intercalating CNTs not only prevent stacking of graphene sheets but also facilitate electron and ion transport. Two-step CVD processes have been devised to grow graphene foam covered with CNT matrix (Figure 4) (Zhu et al., 2014). CNT nano-meshes on top of monolithic graphene skeleton further enhance surface area, conductivity, and charge-transfer

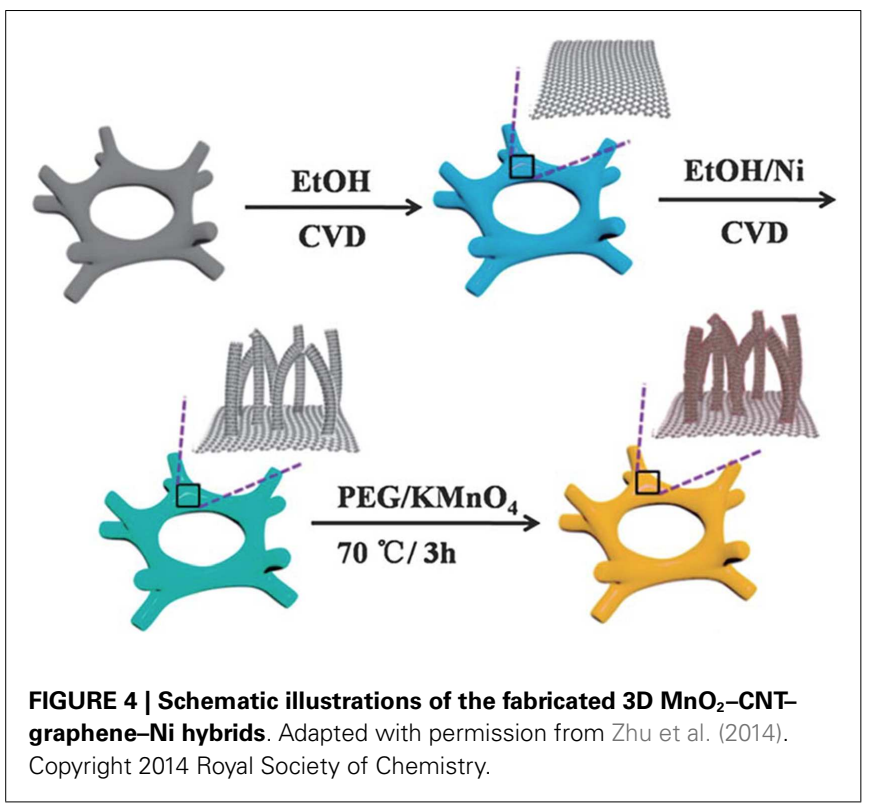

conductance. CNT-graphene hybrid can also simply obtained by dip-coating CNT dispersion on pre-synthesized graphene foam, taking advantage of the strong hydrophobic and pi-pi interactions between CNTs and graphene (Prasad et al., 2014). Fan et al. prepared 3D CNT-graphene sandwich structure from catalyst precursor-coated GO sheets (Ma et al., 2011). 


\section{FUNCTIONALIZATION}

The 3D nanocarbon structures can be functionalized with biomolecules, polymers, or other functional nanomaterials (e.g., precious metal nanoparticles and nanostructured metal oxides) to attain improved or new functionalities (Georgakilas et al., 2012; Chang and Wu, 2013). Functionalization of enzymes via physisorption or covalent bonding renders the 3D nanocarbon bio-catalytic capabilities. Decoration of metal or metal oxide nanoparticles renders high electrochemical or catalytic properties. Integration of conducting polymers enhances the conductivity, mechanical strength, as well as electrochemical activities. The 3D architectures not only provide a large supporting/loading surface area but also alleviate the commonly encountered problem of nanomaterial aggregation. Heteroatom doping is another effective route to modify the properties of 3D nanocarbon structures (Wang et al., 2014).

\section{APPLICATIONS \\ FUEL CELLS}

The 3D CNT/graphene structures have been employed as the electrochemical electrodes for fuel cells that convert chemical energy into electricity (Maiyalagan et al., 2012). In a direct methanol fuel cell, Huang et al. (2014) used Pt-decorated graphene/g- $\mathrm{C}_{3} \mathrm{~N}_{4}$ composite as the anode for methanol oxidation (Figure 5A). It provides higher forward-scan peak current, better anti-poisoning capability, and superior long-term stability than Pt/graphene, $\mathrm{Pt} /$ carbon nitride, and commercial $\mathrm{Pt} / \mathrm{C}$ electrodes. The $3 \mathrm{D}$ porous graphene structure is advantageous for rapid transport of ions, fuel molecules, and electrons, as well as homogeneous and dense loading of Pt nanoparticles. A novel iron nitride/nitrogen dopedgraphene aerogel hybrid synthesized by a two-step hydrothermal process was used as the fuel-cell cathode for oxygen reduction reaction (ORR) (Figure 5B) (Yin et al., 2014). It demonstrated more positive onset reduction potential, higher current

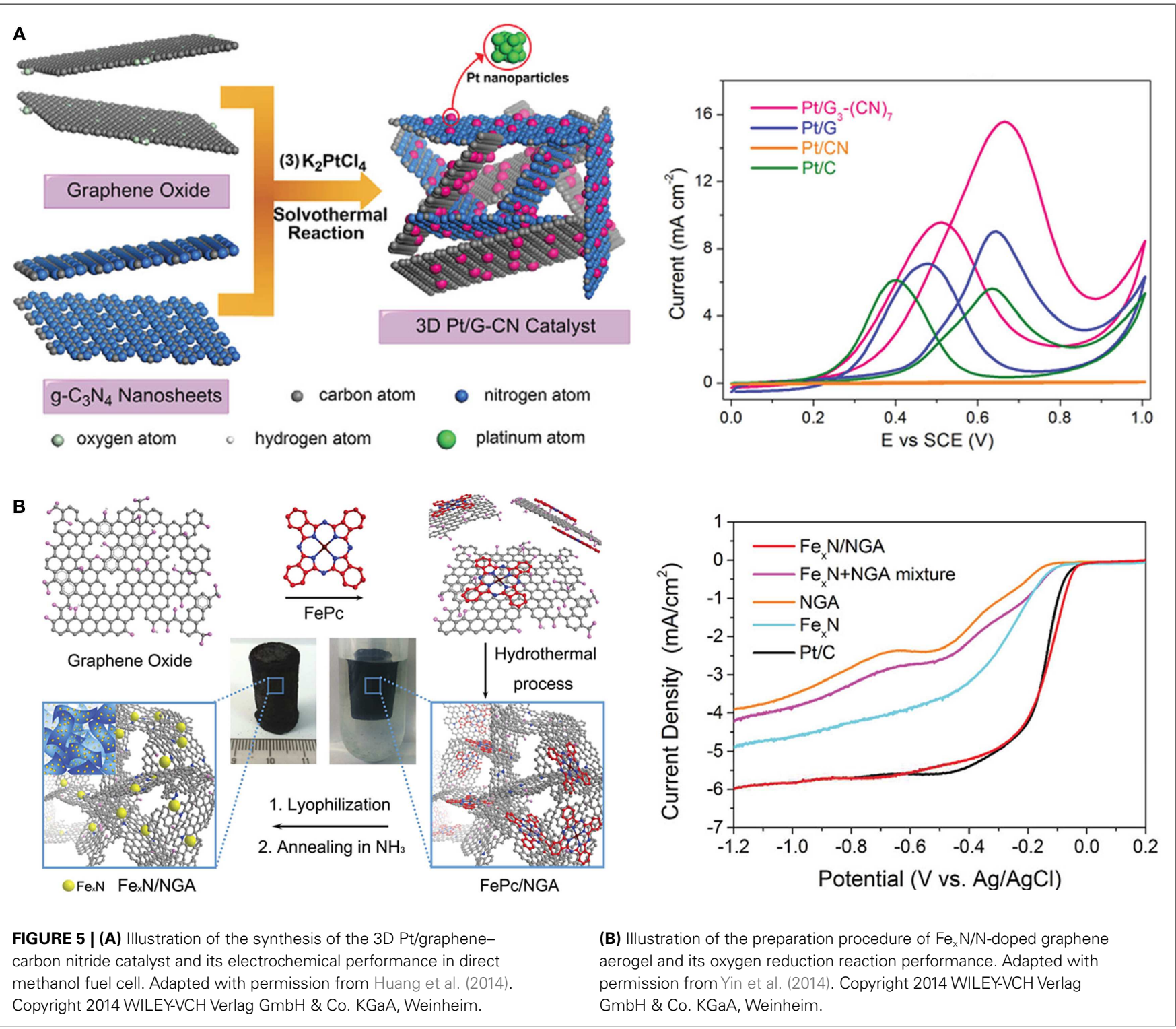


density, and lower charge-transfer resistance than electrodes based on iron nitride or N-doped graphene aerogel. With comparable catalytic activity, the hybrid outperforms the commercial $\mathrm{Pt} / \mathrm{C}$ catalyst in methanol crossover resistance and stability. Heteroatom doping can confer 3D graphene/CNT architectures with remarkable electrocatalytic ability for ORR. The 3D N-doped CNT-arrays have been reported to exhibit excellent ORR activity with the ideal four electron pathway in both acid and alkaline conditions (Gong et al., 2009; Xiong et al., 2010). Using CVD method, Xue et al. (2013) synthesized B/N co-doped graphene foam as efficient metal-free ORR catalyst. It offers a higher reduction peak current than the commercial $\mathrm{Pt}-\mathrm{C} /$ glassy carbon electrode.

\section{BIOFUEL CELLS}

Biofuel cells share the same design with traditional fuel cell while replacing anode catalyst with biological components (enzymes or microorganisms), which are able to harvest biochemical energy into electricity. One major problem of enzymatic biofuel cell is the poor electron transfer from the buried catalytic centers of the enzyme molecules. To tackle this issue, Prasad et al. (2014) employed enzyme decorated 3D hybrid of graphene foam and single-walled CNTs as novel biofuel cell electrodes. The asfabricated device attains the theoretical limit of open circuit voltage $(\sim 1.2 \mathrm{~V})$ and a high power density of $2.27 \mathrm{~mW} \mathrm{~cm}^{-2}$, which benefits from the large surface area and high conductivity of the 3D electrodes, and the nearly perfect electrical coupling ensured by the intimate contact between enzyme molecules and nanotopographic electrode surface. In comparison to the conventional planar electrodes, 3D carbon electrodes can greatly improve the output of microbial fuel cells by promoting colonization and loading of electrogenic bacteria and 3D electrical coupling. Xie et al. (2012) employed 3D CNT-coated macroporous sponge as the bioanode and achieved a maximum current density of $10.63 \mathrm{~mA} \mathrm{~cm}^{-3}$ in glucose media, which was $48 \%$ higher than that of CNT-textile electrode. Yong et al. (2012) demonstrated a novel 3D macroporous anode based on PANI decorated CVDgrown 3D graphene foam, which reached a powder density of $\sim 190 \mathrm{~mW} \mathrm{~m}^{-2}$. It significantly outperforms planar carbon electrodes, e.g., carbon cloth or felt electrode with or without PANI coating.

\section{SUPERCAPACITORS}

The 3D CNT/graphene architectures, with low level aggregation and agglomeration of individual CNTs of graphene sheets, are able to provide large electric double-layer capacitance (EDLC) owing to large surface area. In addition, they permit high loading of electrochemically active nanomaterials to gain high electrochemical pseudocapacitance. The 3D carbon nanocup-CNT structures have been synthesized on anodized aluminum oxide for EDLC based supercapacitors (Hahm et al., 2012). The CNT modification gives carbon nanocup an extremely high-specific surface area $\left(1340 \mathrm{~m}^{2} \mathrm{~g}^{-1}\right)$ and enhances its capacitance to $45 \mathrm{Fg}^{-1}$ in $1 \mathrm{M}$ $\mathrm{LiPF}_{6}$. Open-porous rGO foam prepared from autoclave leavening GO film is also able to provide a large EDLC $\left(110 \mathrm{Fg}^{-1}\right)$ which is one order higher than that of $\mathrm{rGO}$ films $\left(17 \mathrm{Fg}^{-1}\right)$ (Niu et al., 2012). Seamless and $\mathrm{sp}^{2}$-carbon connection of CVD grown graphene are advantageous to exploit the synergistic effects between CNT and graphene. The 3D graphene-CNT hybrids grown on nickel foam prepared by two-step CVD approach have been reported to exhibit a five-time-higher specific capacitance than CNT directly grown on inconel without graphene as the supporting substrate (Yan et al., 2013).

Jiang et al. (2013) prepared vertically aligned CNT forest with uniformly embedded nickel oxide nanoparticles, which delivered a 5.7 times higher specific capacitance $\left(1.26 \mathrm{~F} \mathrm{~cm}^{-3}\right)$ than pure $\mathrm{CNT}$ forest samples because of combination of both EDLC and pseduocapacitance (Figure 6A). Niu et al. (2014) reported a general strategy to prepare functional porous graphene hybrid architectures (Figure 6B). As an example, rGO/PANI nanofiber electrode offers a high-specific capacitance of $475 \mathrm{~F} \mathrm{~g}^{-1}$, much larger than that of pure rGO architecture. CVD-grown graphene foam with well-defined macroporous structure can serve as an excellent platform to prepare various functional supercapacitor electrodes (Dong et al., 2012a,d). For example, deposition of nanostructured $\mathrm{Co}_{3} \mathrm{O}_{4}$ on CVD-graphene foam offers a high-specific capacitance of $1100 \mathrm{Fg}^{-1}$ (Dong et al., 2012f). Zhu et al. prepared 3D $\mathrm{MnO}_{2}-$ CNT-graphene-nickel hybrid foam. Such porous and highly conductive electrode delivers a specific capacitance of $\sim 251 \mathrm{Fg}^{-1}$ (Zhu et al., 2014). Heteroatom doping and integration of CNTs can be employed to enhance the capacitive behavior. You et al. (2013) prepared N-doped 3D CNT-graphene aerogel electrode for supercapacitor application. It gives a maximum specific capacitance of $180 \mathrm{Fg}^{-1}$ at a current density of $0.5 \mathrm{Ag}^{-1}$. Addition of CNTs effectively prevents graphene aggregation and improves the conductivity.

\section{LITHIUM ION BATTERIES}

The $3 \mathrm{D}$ porous graphene/CNT architectures are also attractive for lithium ion batter (LIB), which can improve Li ion diffusion and tolerance to mechanical stress induced by ion deintercalation process. Ruoff group first applied CVD-grown 3D graphene for LIB cathode (Ji et al., 2012). Accounting the total mass, such 3D graphene loaded with lithium iron phosphate exhibited a 23 and $170 \%$ higher specific capacity than that of $\mathrm{Al}$ - and Ni-foam loaded with lithium iron phosphate, respectively. Xiao et al. (2013) prepared 3D graphene aerogel/ $/ \mathrm{Fe}_{2} \mathrm{O}_{3}$ composite by a one-pot hydrothermal process. The synergistic interaction between uniformly dispersed $\mathrm{Fe}_{2} \mathrm{O}_{3}$ nanoparticles and robust $3 \mathrm{D}$ graphene aerogel endows such electrode with a high reversible capacity of $995 \mathrm{~mA} \mathrm{hg}^{-1}$, which is almost four times to that of physically mixed $\mathrm{Fe}_{2} \mathrm{O}_{3}$-graphene electrode. Integration of CNTs to a similar electrode further increases the reversible capacity to $1118 \mathrm{~mA} \mathrm{hg}^{-1}$ (Liu et al., 2013a). Qiu et al. (2014) prepared $\mathrm{TiO}_{2}$ nanocrystal/graphene aerogel as LIB anode, using glucose as dispersion and linking agent. A reversible capacity of 956.2 $\mathrm{mA} \mathrm{h} \mathrm{g}^{-1}$ was achieved, which is superior to the previously reported $\mathrm{TiO}_{2}$ /carbon electrodes (Yang et al., 2012). Even at a high current density of $5000 \mathrm{mAhg}^{-1}$, the hybrid foam electrode still gives a reversible capacity of $99 \mathrm{~mA} \mathrm{~h} \mathrm{~g}^{-1}$, four times higher than that of pure $\mathrm{TiO}_{2}$ based electrode. Gong et al. (2014) developed a bottom-up method to construct 3D LIB electrode from GO sheets and exfoliated $\mathrm{MoS}_{2}$ nanosheets. The resultant $\mathrm{MoS}_{2}$-graphene electrodes exhibit a high reversible capacity of 


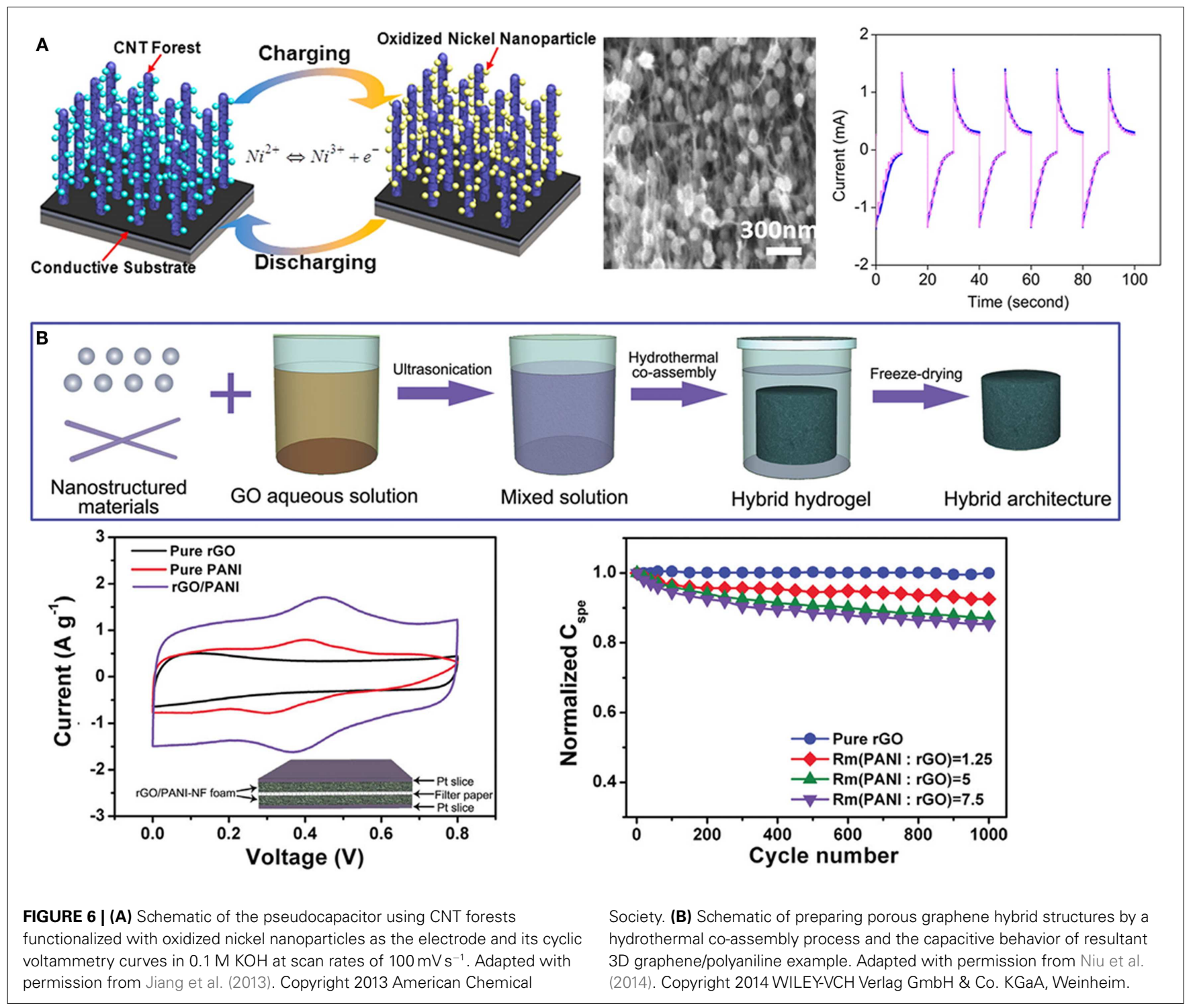

$\sim 1200 \mathrm{~mA} \mathrm{~h} \mathrm{~g}^{-1}$, good stability, and excellent rate performance. Cao et al. (2013) coated nanostructured $\mathrm{MoS}_{2}$ on CVD-grown graphene foam by dip-coating and annealing method and demonstrated a high reversible capacity of $877 \mathrm{~mA} \mathrm{hg}^{-1}$ at a current density of $100 \mathrm{~mA} \mathrm{~h}^{-1}$.

\section{CONCLUSION AND PERSPECTIVES}

In summary, electrochemical electrodes based on 3D nanocarbon structures, as compared with conventional planar electrodes, can greatly enhance the capacity, efficiency, or stability for the energy storage and conversion devices. Although this article focuses on energy devices, electrochemical electrodes based on $3 \mathrm{D}$ architecture of CNTs or graphene or their hybrid are also instrumental to other applications. For example, Dong et al. (2012b) developed a hybrid foam of graphene and CNT for selective removal of oil and organic solvent from water surface. Yin et al. (2013) used 3D graphene/metal oxide composite for effective capacitive deionization from water. Ananthanarayanan et al. (2014) successfully synthesized fluorescent graphene quantum dots from 3D graphene foam. Furthermore, electrochemical sensors based on 3D nanocarbon electrodes offer higher sensitivity or larger detection range as compared to traditional planar electrodes (Dong et al., 2012c,e; Xi et al., 2013). The 3D nanocarbon substrates can also serve as supporting scaffolds to incorporate other $2 \mathrm{D}$ materials such as transition metal dichalcogenide nanosheets. Although with challenges, developing new strategies to controllably fabricate functional 3D electrodes while preserving the intrinsic merits of CNTs or graphene will continue to provide tremendous thrusts to energy as well as many other applications.

\section{ACKNOWLEDGMENTS}

We thank the support from the Agency for Science, Technology and Research (A*STAR) under a SERC Grant (102 170 0142) and Ministry of Education of Singapore under an AcRF Tier 2 grant (MOE2011-T2-2-010). 


\section{REFERENCES}

Ananthanarayanan, A., Wang, X. W., Routh, P., Sana, B., Lim, S., Kim, D. H., et al. (2014). Facile synthesis of graphene quantum dots from 3D graphene and their application for $\mathrm{Fe}^{3+}$ sensing. Adv. Funct. Mater. 24, 3021-3026. doi:10.1002/adfm.201303441

Cao, X. H., Shi, Y. M., Shi, W. H., Rui, X. H., Yan, Q. Y., Kong, J., et al. (2013). Preparation of $\mathrm{MoS}_{2}$-coated three-dimensional graphene networks for highperformance anode material in lithium-ion batteries. Small 9, 3433-3438. doi:10.1002/smll.201202697

Chang, H. X., and Wu, H. K. (2013). Graphene-based nanocomposites: preparation, functionalization, and energy and environmental applications. Energy Environ. Sci. 6, 3483-3507. doi:10.1039/C3ee42518e

Chen, Z. P., Ren, W. C., Gao, L. B., Liu, B. L., Pei, S. F., and Cheng, H. M. (2011). Threedimensional flexible and conductive interconnected graphene networks grown by chemical vapour deposition. Nat. Mater. 10, 424-428. doi:10.1038/Nmat3001

Dong, X. C., Cao, Y. F., Wang, J., Chan-Park, M. B., Wang, L. H., Huang, W., et al. (2012a). Hybrid structure of zinc oxide nanorods and three dimensional graphene foam for supercapacitor and electrochemical sensor applications. RSC Adv. 2, 4364-4369. doi:10.1039/C2ra01295b

Dong, X. C., Chen, J., Ma, Y. W., Wang, J., Chan-Park, M. B., Liu, X. M., et al. (2012b). Superhydrophobic and superoleophilic hybrid foam of graphene and carbon nanotube for selective removal of oils or organic solvents from the surface of water. Chem. Commun. 48, 10660-10662. doi:10.1039/C2cc35844a

Dong, X. C., Ma, Y. W., Zhu, G. Y., Huang, Y. X., Wang, J., Chan-Park, M. B., et al. (2012c). Synthesis of graphene-carbon nanotube hybrid foam and its use as a novel three-dimensional electrode for electrochemical sensing. J. Mater. Chem. 22, 17044-17048. doi:10.1039/C2jm33286h

Dong, X. C., Wang, J. X., Wang, J., Chan-Park, M. B., Li, X. G., Wang, L. H., et al. (2012d). Supercapacitor electrode based on three-dimensional graphene-polyaniline hybrid. Mater. Chem. Phys. 134, 576-580. doi:10.1016/ j.matchemphys.2012.03.066

Dong, X. C., Wang, X. W., Wang, L. H., Song, H., Zhang, H., Huang, W., et al. (2012e). 3D graphene foam as a monolithic and macroporous carbon electrode for electrochemical sensing. ACS Appl. Mater. Interfaces 4, 3129-3133. doi:10.1021/Am300459m

Dong, X. C., Xu, H., Wang, X. W., Huang, Y. X., Chan-Park, M. B., Zhang, H., et al. (2012f). 3D Graphene-cobalt oxide electrode for high-performance supercapacitor and enzymeless glucose detection. ACS Nano 6, 3206-3213. doi:10.1021/Nn300097q

Georgakilas, V., Otyepka, M., Bourlinos, A. B., Chandra, V., Kim, N., Kemp, K. C., et al. (2012). Functionalization of graphene: covalent and non-covalent approaches, derivatives and applications. Chem. Rev. 112, 6156-6214. doi:10. $1021 / \mathrm{cr} 3000412$

Gong, K. P., Du, F., Xia, Z. H., Durstock, M., and Dai, L. M. (2009). Nitrogen-doped carbon nanotube arrays with high electrocatalytic activity for oxygen reduction. Science 323, 760-764. doi:10.1126/science.1168049

Gong, Y. J., Yang, S. B., Zhan, L., Ma, L. L., Vajtai, R., and Ajayan, P. M. (2014). A bottom-up approach to build 3D architectures from nanosheets for superior lithium storage. Adv. Funct. Mater. 24, 125-130. doi:10.1002/adfm.201300844

Hahm, M. G., Reddy, A. L. M., Cole, D. P., Rivera, M., Vento, J. A., Nam, J., et al. (2012). Carbon nanotube-nanocup hybrid structures for high power supercapacitor applications. Nano Lett. 12, 5616-5621. doi:10.1021/Nl3027372

Hu, C. G., Zhai, X. Q., Liu, L. L., Zhao, Y., Jiang, L., and Qu, L. T. (2013). Spontaneous reduction and assembly of graphene oxide into three-dimensional graphene network on arbitrary conductive substrates. Sci. Rep. 3, 2065. doi:10.1038/Srep02065

Huang, H. J., Yang, S. B., Vajtai, R., Wang, X., and Ajayan, P. M. (2014). Pt-decorated $3 \mathrm{D}$ architectures built from graphene and graphitic carbon nitride nanosheets as efficient methanol oxidation catalysts. Adv. Mater. doi:10.1002/adma.201401877

Jariwala, D., Sangwan, V. K., Lauhon, L. J., Marks, T. J., and Hersam, M. C. (2013). Carbon nanomaterials for electronics, optoelectronics, photovoltaics, and sensing. Chem. Soc. Rev. 42, 2824-2860. doi:10.1039/C2cs35335k

Ji, H. X., Zhang, L. L., Pettes, M. T., Li, H. F., Chen, S. S., Shi, L., et al. (2012). Ultrathin graphite form: a three-dimensional conductive network for battery electrode. Nano Lett. 12, 2446-2451. doi:10.1021/nl300528p

Jiang, X., Ma, Y. W., Li, J. J., Fan, Q. L., and Huang, W. (2010). Self-assembly of reduced graphene oxide into three-dimensional architecture by divalent ion linkage. J. Phys. Chem. C 114, 22462-22465. doi:10.1021/jp108081g
Jiang, Y. Q., Wang, P. B., Zang, X. N., Yang, Y., Kozinda, A., and Lin, L. W. (2013). Uniformly embedded metal oxide nanoparticles in vertically aligned carbon nanotube forests as pseudocapacitor electrodes for enhanced energy storage. Nano Lett. 13, 3524-3530. doi:10.1021/nl400921p

Li, W., Gao, S., Wu, L., Qiu, S. Q., Guo, Y. F., Geng, X. M., et al. (2013). High-density three-dimension graphene macroscopic objects for high-capacity removal of heavy metal ions. Sci. Rep. 3, 2125. doi:10.1038/srep02125

Liu, J. L., Jiang, J. B., Qian, D., Tan, G. R., Peng, S. J., Yuan, H. M., et al. (2013a). Facile assembly of a $3 \mathrm{D} \mathrm{rGO} / \mathrm{MWCNTs} / \mathrm{Fe}_{2} \mathrm{O}_{3}$ ternary composite as the anode material for high-performance lithium ion batteries. RSC Adv. 3, 15457-15466. doi:10.1039/C3ra42780c

Liu, Y. F., Ba, H., Nguyen, D. L., Ersen, O., Romero, T., Zafeiratos, S., et al. (2013b). Synthesis of porous carbon nanotubes foam composites with a high accessible surface area and tunable porosity. J. Mater. Chem. A 1, 9508-9516. doi:10.1039/C3TA10695K

Liu, Y. X., Dong, X. C., and Chen, P. (2012). Biological and chemical sensors based on graphene materials. Chem. Soc. Rev. 41, 2283-2307. doi:10.1039/C1CS15270J

Ma, Y. W., Sun, L. Y., Huang, W., Zhang, L. R., Zhao, J., Fan, Q. L., et al. (2011). Three-dimensional nitrogen-doped carbon nanotubes/graphene structure used as a metal-free electrocatalyst for the oxygen reduction reaction. J. Phys. Chem. C 115, 24592-24597. doi:10.1021/Jp207736h

Maiyalagan, T., Dong, X. C., Chen, P., and Wang, X. (2012). Electrodeposited Pt on three-dimensional interconnected graphene as a free-standing electrode for fuel cell application. J. Mater. Chem. 22, 5286-5290. doi:10.1039/C2jm16541d

Niu, Z. Q., Chen, J., Hng, H. H., Ma, J., and Chen, X. D. (2012). A leavening strategy to prepare reduced graphene oxide foams. Adv. Mater. 24, 4144-4150. doi:10.1002/adma.201200197

Niu, Z. Q., Liu, L. L., Zhang, L., Shao, Q., Zhou, W. Y., Chen, X. D., et al. (2014). A universal strategy to prepare functional porous graphene hybrid architectures. Adv. Mater. 26, 3681-3687. doi:10.1002/adma.201400143

Peng, Q. Y., Li, Y. B., He, X. D., Gui, X. C., Shang, Y. Y., Wang, C. H., et al. (2014). Graphene nanoribbon aerogels unzipped from carbon nanotube sponges. $A d v$. Mater. 26, 3241-3247. doi:10.1002/adma.201305274

Prasad, K. P., Chen, Y., and Chen, P. (2014). Three-dimensional graphene-carbon nanotube hybrid for high-performance enzymatic biofuel cells. Acs Appl. Mater. Interfaces 6, 3387-3393. doi:10.1021/Am405432b

Qiu, B. C., Xing, M. Y., and Zhang, J. L. (2014). Mesoporous $\mathrm{TiO}_{2}$ nanocrystals grown in situ on graphene aerogels for high photocatalysis and lithium-ion batteries. J. Am. Chem. Soc. 136, 5852-5855. doi:10.1021/Ja500873u

Ren, G. F., Pan, X., Bayne, S., and Fan, Z. Y. (2014). Kilohertz ultrafast electrochemical supercapacitors based on perpendicularly-oriented graphene grown inside of nickel foam. Carbon N. Y. 71, 94-101. doi:10.1016/j.carbon.2014.01.017

Shan, C. S., Zhao, W. J., Lu, X. L., O’brien, D. J., Li, Y. P., Cao, Z. Y., et al. (2013). Three-dimensional nitrogen-doped multiwall carbon nanotube sponges with tunable properties. Nano Lett. 13, 5514-5520. doi:10.1021/Nl403109g

Sun, G. Z., Pan, Y. Z., Zhan, Z. Y., Zheng, L. X., Lu, J. Y., Pang, J. H. L., et al. (2011a). Reliable and large curvature actuation from gradient-structured graphene oxide. J. Phys. Chem. C 115, 23741-23744. doi:10.1021/Jp207986m

Sun, Y. Q., Wu, Q. O., and Shi, G. Q. (2011b). Graphene based new energy materials. Energy Environ. Sci. 4, 1113-1132. doi:10.1039/C0ee00683a

Sun, G. Z., Zheng, L. X., Zhan, Z. Y., Zhou, J. Y., Liu, X. B., and Li, L. (2014). Actuation triggered exfoliation of graphene oxide at low temperature for electrochemical capacitor applications. Carbon N. Y. 68, 748-754. doi:10.1016/j.carbon. 2013.11.063

Wang, X. B., Zhang, Y. J., Zhi, C. Y., Wang, X., Tang, D. M., Xu, Y. B., et al. (2013). Three-dimensional strutted graphene grown by substrate-free sugar blowing for high-power-density supercapacitors. Nat. Commun. 4, 2905. doi:10.1038/ ncomms3905

Wang, X. W., Sun, G. Z., Routh, P., Kim, D. H., Huang, W., and Chen, P. (2014). Heteroatom-doped graphene materials: syntheses, properties and applications. Chem. Soc. Rev. doi:10.1039/C4CS00141A

Weiss, N. O., Zhou, H. L., Liao, L., Liu, Y., Jiang, S., Huang, Y., et al. (2012). Graphene: an emerging electronic material. Adv. Mater. 24, 5782-5825. doi:10.1002/adma. 201201482

Xi, F. N., Zhao, D. J., Wang, X. W., and Chen, P. (2013). Non-enzymatic detection of hydrogen peroxide using a functionalized three-dimensional graphene electrode. Electrochem. commun. 26, 81-84. doi:10.1016/j.elecom.2012.10.017 
Xiao, L., Wu, D. Q., Han, S., Huang, Y. S., Li, S., He, M. Z., et al. (2013). Selfassembled $\mathrm{Fe}_{2} \mathrm{O}_{3}$ /graphene aerogel with high lithium storage performance. ACS Appl. Mater. Interfaces 5, 3764-3769. doi:10.1021/Am400387t

Xie, X., Ye, M., Hu, L. B., Liu, N., Mcdonough, J. R., Chen, W., et al. (2012). Carbon nanotube-coated macroporous sponge for microbial fuel cell electrodes. Energy Environ. Sci. 5, 5265-5270. doi:10.1039/C1ee02122b

Xie, X., Zhou, Y. L., Bi, H. C., Yin, K. B., Wan, S., and Sun, L. T. (2013). Largerange control of the microstructures and properties of three-dimensional porous graphene. Sci. Rep. 3, 2117. doi:10.1038/Srep02117

Xiong, W., Du, F., Liu, Y., Perez, A., Supp, M., Ramakrishnan, T. S., et al. (2010). 3-D Carbon nanotube structures used as high performance catalyst for oxygen reduction reaction. J. Am. Chem. Soc. 132, 15839-15841. doi:10.1021/ ja104425h

Xu, Y. X., Sheng, K. X., Li, C., and Shi, G. Q. (2010). Self-assembled graphene hydrogel via a one-step hydrothermal process. ACS Nano 4, 4324-4330. doi:10.1021/ nn $101187 \mathrm{z}$

Xue, Y. H., Yu, D. S., Dai, L. M., Wang, R. G., Li, D. Q., Roy, A., et al. (2013). Three-dimensional B,N-doped graphene foam as a metal-free catalyst for oxygen reduction reaction. Phys. Chem. Chem. Phys. 15, 12220-12226. doi:10.1039/ C3cp51942b

Yan, Z., Ma, L. L., Zhu, Y., Lahiri, I., Hahm, M. G., Liu, Z., et al. (2013). Threedimensional metal-graphene-nanotube multifunctional hybrid materials. ACS Nano 7, 58-64. doi:10.1021/nn3015882

Yang, S., Cai, Y., Cheng, Y. W., Varanasi, C. V., and Liu, J. (2012). Monolithic coaerogels of carbon/titanium dioxide as three dimensional nanostructured electrodes for energy storage. J. Power Sources 218, 140-147. doi:10.1016/j.jpowsour. 2012.06.070

Yang, Z. Y., Jin, L. J., Lu, G. Q., Xiao, Q. Q., Zhang, Y. X., Jing, L., et al. (2014). Sponge-templated preparation of high surface area graphene with ultrahigh capacitive deionization performance. Adv. Funct. Mater. 24, 3917-3925. doi:10.1002/adfm.201304091

Yin, H., Zhang, C. Z., Liu, F., and Hou, Y. L. (2014). Hybrid of iron nitride and nitrogen-doped graphene aerogel as synergistic catalyst for oxygen reduction reaction. Adv. Funct. Mater. 24, 2930-2937. doi:10.1002/adfm.201303902

Yin, H. J., Zhao, S. L., Wan, J. W., Tang, H. J., Chang, L., He, L. C., et al. (2013). Three-dimensional graphene/metal oxide nanoparticle hybrids for highperformance capacitive deionization of saline water. Adv. Mater. 25, 6270-6276. doi:10.1002/adma.201302223

Yong, Y. C., Dong, X. C., Chan-Park, M. B., Song, H., and Chen, P. (2012). Macroporous and monolithic anode based on polyaniline hybridized three-dimensional graphene for high-performance microbial fuel cells. ACS Nano 6, 2394-2400. doi:10.1021/Nn204656d

You, B., Wang, L. L., Yao, L., and Yang, J. (2013). Three dimensional N-doped graphene-CNT networks for supercapacitor. Chem. Commun. 49, 5016-5018. doi:10.1039/C3cc41949e

Zhan, Z. Y., Zhang, Y. N., Sun, G. Z., Zheng, L. X., and Liao, K. (2011). The effects of catalyst treatment on fast growth of millimeter-long multi-walled carbon nanotube arrays. Appl. Surf. Sci. 257, 7704-7708. doi:10.1016/j.apsusc.2011.04.013

Zhang, Q., Huang, J. Q., Qian, W. Z., Zhang, Y. Y., and Wei, F. (2013). The road for nanomaterials industry: a review of carbon nanotube production, posttreatment, and bulk applications for composites and energy storage. Small 9, 1237-1265. doi:10.1002/smll.201203252

Zhao, D. D., Yang, Z., Zhang, L. Y., Feng, X. L., and Zhang, Y. F. (2011). Electrodeposited manganese oxide on nickel foam-supported carbon nanotubes for electrode of supercapacitors. Electrochem. Solid State Lett. 14, A93-A96. doi:10.1149/1.3562927

Zheng, L. X., Sun, G. Z., and Zhan, Z. Y. (2010). Tuning array morphology for highstrength carbon-nanotube fibers. Small 6, 132-137. doi:10.1002/smll.200900954

Zhu, G. Y., He, Z., Chen, J., Zhao, J., Feng, X. M., Ma, Y. W., et al. (2014) Highly conductive three-dimensional $\mathrm{MnO}_{2}$-carbon nanotube-graphene-Ni hybrid foam as a binder-free supercapacitor electrode. Nanoscale 6, 1079-1085. doi:10.1039/C3NR04495E

Conflict of Interest Statement: The authors declare that the research was conducted in the absence of any commercial or financial relationships that could be construed as a potential conflict of interest.

Received: 15 July 2014; paper pending published: 28 July 2014; accepted: 04 August 2014; published online: 20 August 2014.

Citation: Wang X, Sun G and Chen P (2014) Three-dimensional porous architectures of carbon nanotubes and graphene sheets for energy applications. Front. Energy Res. 2:33. doi: $10.3389 /$ fenrg.2014.00033

This article was submitted to Nanoenergy Technologies and Materials, a section of the journal Frontiers in Energy Research.

Copyright (C) 2014 Wang, Sun and Chen. This is an open-access article distributed under the terms of the Creative Commons Attribution License (CC BY). The use, distribution or reproduction in other forums is permitted, provided the original author(s) or licensor are credited and that the original publication in this journal is cited, in accordance with accepted academic practice. No use, distribution or reproduction is permitted which does not comply with these terms. 